\title{
Fipronil induces lung inflammation in vivo and cell death in vitro
}

\author{
Kaitlin Merkowsky ${ }^{1}$, Ram S. Sethi ${ }^{2}$, Jatinder P. S. Gill ${ }^{3}$ and Baljit Singh ${ }^{1 *}$
}

\begin{abstract}
Background: Fipronil is an insecticide that acts at the gamma-aminobutyric acid receptor and glutamate-gated chloride channels in the central nervous systems of target organisms. The use of fipronil is increasing across the globe. Presently, very little data exist on the potential impact of exposure to fipronil on the lungs.

Methods: We studied effects of intranasal $(N=8)$ and oral $(N=8)$ treatment with fipronil $(10 \mathrm{mg} / \mathrm{kg})$ on lungs of mice. Control mice were given groundnut oil orally $(N=7)$ or ethanol intranasally $(N=7)$ as these were the vehicles for respective treatments.

Results: Hematoxylin-eosin stained lung sections showed normal histology in the control lungs compared to the thickened alveolar septa, disruption of the airways epithelium and damage to vascular endothelium in the intranasal and the oral groups. Mice exposed to fipronil either orally or intranasally showed increased von Willebrand factor staining in the endothelium and septal capillaries. Compared to the control mice, TLR4 expression in airway epithelium was increased in mice treated intranasally but not orally with fipronil. Oral fipronil reduced TLR9 staining in the airway epithelium but intranasal exposure caused intense staining in the alveolar septa and airway epithelium. There were higher numbers of TLR4 positive cells in alveolar septa in lungs of mice treated intranasally $(P=0.010)$ compared to the respective control and orally treated mice but no significant differences between treatments for TLR9 positive stained cells $(P=0.226)$. The U937 macrophage cells exposed to fipronil at concentrations of $0.29 \mu \mathrm{m}$ to $5.72 \mu \mathrm{m} / \mathrm{ml}$ over 3 - or 24-hour showed significant increase in cell death at higher concentrations of fipronil $(P<0.0001)$. Western blots revealed no effect of fipronil on TLR4 $(P=0.49)$ or TLR9 $(P=0.94)$ expression on macrophage cell line.
\end{abstract}

Conclusion: While both oral or intranasal fipronil treatments induced signs of lung inflammation, the number TLR4-positive septal cells was increased only following intranasal treatment. Fipronil causes macrophage cell death without altering TLR4 and TLR9 expression in vitro.

Keywords: Macrophage, Pesticides, Lung Inflammation, TLR4, TLR9

\section{Background}

The Sumerians were probably the first to used elemental sulphur against insects and mites in $2500 \mathrm{BC}$ and ancient Chinese cultures treated body lice with arsenic and mercury. While the ingenuity of these ancient cultures can be appreciated, it is unlikely arsenic would be embraced today as an effective pesticide due to its deleterious effects on human health. The most prominent example in modern times of the struggle between insect control and public health concerns is

\footnotetext{
* Correspondence: baljit.singh@usask.ca

'Department of Veterinary Biomedical Sciences, Western College of Veterinary Medicine, University of Saskatchewan, 52 Campus Drive, Saskatoon, SK S7N 5B4, Canada

Full list of author information is available at the end of the article
}

dichlorodiphenyltrichloroethane (DDT), which belongs to organochlorine group of pesticides [1], and showed unparalleled effectiveness in bringing down cases of malaria. While pesticides and insecticides are the tools to manage pests and insects, many health effects of these chemicals have also be recorded.

Fipronil (( \pm )-5-amino-1- (2,6-dichloro- $\alpha, \alpha, \alpha$-trifluorop-tolyl)- 4-trifluoromethylsulfinylpyrazole-3-carbonitrile) belongs to the phenylpyrazole family and is extensively used around the world in various anti-flea and tick sprays and for pest control in agriculture [2]. Fipronil acts as an antagonist at GABA-gated chloride channels but has a higher affinity for these channels in insects compared to non-target organisms such as humans, making it a 
seemingly safer product in these regards [3-5]. Fipronil is metabolized into many metabolites including sulfone, sulphide, and desulfinyl. However, it has been demonstrated the primary metabolite of fipronil, fipronil sulfone, actually has a much greater affinity for these channels in mammals than those in insects indicating potential detrimental effects of the break-down products to non-target organisms [6]. One of the studies found that following a single oral exposure fipronil sulfone persists for much longer duration than fipronil in high fat containing tissues especially adipose tissue, adrenals and the liver which leads to bioamplification along the food chain $[7,8]$. It has been shown that both sulfone and desulfinyl are potent mitochondrial uncouplers and calcium efflux inducers but may differ in their potencies [9]. Nevertheless, exposure to the parent compound is pre-requisite for the generation of fipronil metabolites. Combined subchronic exposure to fipronil and fluoride induces biochemical alterations in buffalo calves $[10,11]$. Fipronil also induces oxidative stress and activation of MAPK, induction of apoptosis via caspase- 9 and caspase3 , and reduction in differentiated cell numbers [12-14]. During an examination of effects of fipronil on liver p450 enzymes, it was found that fipronil is an inducer of hepatic phase I CYP enzymes that may increase potential for interactions with xenobiotics [15]. While recent studies have shown the toxic effects of fipronil on liver especially the mitochondria in liver cells $[9,16]$, there currently are no data on the pulmonary effects of fipronil in animals.

This study was designed to investigate the pulmonary effects of fipronil following oral or intranasal exposures through testing of hypothesis that exposure to low levels of fipronil will induce lung inflammation and cell death. The inhalation route remains a major route of exposure in developing countries where agricultural workers generally spray pesticides without appropriate personal protective gear. The oral route occurs through contamination of food and water with pesticides. We also investigated the effects of fipronil on a macrophage cell line in vitro. The data show fipronil causes cell death in vitro and induces lung inflammation following both oral and intranasal routes of administration but increases number of TLR4 cells only after intranasal treatment.

\section{Methods}

\section{In vivo experiments}

Experiment was conducted following approval from the Institutional Animal Ethics Committee (IAEC), Guru Angad Dev Veterinary and Animal Sciences University, Ludhiana. Swiss albino mice, ages $8-10$ weeks, were housed in laboratory animal cages at $18-22{ }^{\circ} \mathrm{C}$ and $12: 12$ light-dark cycles. Mice had access to feed (Ashirwad Industries, Chandigarh, Punjab, India) and water ad libitum.

\section{Experiment design}

An initial experiment was first conducted where mice $(n=$ 8) were exposed to $8 \mathrm{mg} / \mathrm{kg}$ or $2 \mathrm{mg} / \mathrm{kg}$ of fipronil via intranasal or oral routes $(n=2$ each) or treated with respective vehicles $(n=2$ each for ethanol for intransal and corn oil for oral route) to determine an appropriate dose of fipronil (Sigma-Aldrich S. Louis, USA; <=100 \% purity). Based on the preliminary study, we decided on $8 \mathrm{mg} / \mathrm{kg}$ of fipronil as the dose for both intranasal and oral exposure routes. This dose was determined as it was $10 \%$ of the oral $\mathrm{LD}_{50}$ for mice to reduce chances of acute toxicity and death and treated mice for seven days to induce sub-chronic toxic effects (http://npic.orst.edu/factsheets/fiptech.pdf).

Control mice $(N=7)$ received groundnut oil, which was used as a vehicle for treating mice $(N=8)$ orally with fipronil $(8 \mathrm{mg} / \mathrm{kg} /$ day $)$. Mice $(N=8)$ were treated intranasally with fipronil $(8 \mathrm{mg} / \mathrm{kg} /$ day $)$ in dissolved in ethanol. Control mice $(N=7)$ received intranasal treatment ethanol. Unfortunately the intranasal groups of mice experienced high mortality following anaesthesia and the ketamine/xylazine dose was adjusted daily to reduce chances of mortality in the mice.

After 7 days mice were euthanized with a lethal dose of ketamine/xylazine $(0.1 \mu \mathrm{l} / 10 \mathrm{~g}$ of body weight $)$ and cardiac puncture was done to collect blood. The trachea was isolated and a small cannula was inserted to perform lung lavage. Lungs were lavaged 3 times with $0.5 \mathrm{ml}$ of phosphate buffered saline to collect broncho-alveolar lavage (BAL) fluid. The left lung was fixed in $10 \%$ formalin overnight $(24 \mathrm{~h})$. Lungs were placed in filter capsules and processed by the acetone and benzene method to obtain $5 \mu \mathrm{m}$ thick paraffin sections.

\section{Hematoxylin and eosin staining}

The paraffin sections of lungs from all groups were stained with H\&E staining for routine histopathology.

\section{Immunohistochemistry}

The immunohistochemistry was performed as reported earlier (Sethi 2013). Briefly tissue sections were de-paraffinized and rehydrated. The tissue peroxidases were inactivated with $0.5 \% \mathrm{H}_{2} \mathrm{O}_{2}$ in methanol for $20 \mathrm{~min}$. Pepsin $(2 \mathrm{mg} / \mathrm{ml}$ in $0.01 \mathrm{~N} \mathrm{HCl})$ was used to unmask antigen-binding sites $(60 \mathrm{~min})$ and then $1 \%$ bovine serum albumin (BSA) in PBS was used to prevent non-specific binding (30 min). Next the tissues were incubated overnight $(16 \mathrm{~h})$ at $4{ }^{\circ} \mathrm{C}$ with the following antibodies: von Willebrand Factor (1:500, DAKO A0082), Toll-Like Receptor 4 (1:25, IMG-578A, IMGENEX) and Toll-Like Receptor 9 (1:50, IMG-3051, IMGENEX) followed by appropriate secondary antibody (vWF at 1:300 and TLR at 1:100, all from DAKO). VECTOR VIP Peroxidase Substrate Kit (Vector laboratories, Burlingame, 
CA) was used for colour-development ed followed by counter staining with methyl green (Vector laboratories).

\section{Grading for immunohistochemistry}

Lungs sample from all the animals were used for grading of immunohistochemical staining intensity and quantification of the number of TLR4 or TLR9 positive cells in the alveolar septa (Table B). For each tissue section five random fields of vision was assessed and a score of 1-4 ( 1 being least intense, 4 being most intense) was assigned for each of the criteria. The cells were counted in 5 consecutive fields under 100X. The sections were graded depending on the staining intensity of vWF in the large blood vessels, or TLR4 or TLR9 in the bronchial epithelium and continuity of the airways epithelium as interrupted or intact (Table B). One individual who was blinded to what treatments of each animal performed the scoring.

\section{In vitro experiments}

U937 cell line was obtained from ATCC ${ }^{\circ}$. RPMI Complete Media $+10 \%$ Fetal Bovine Serum (FBS) (Gibco) was warmed to $37^{\circ} \mathrm{C}$. Aliquots of cells were thawed in a hot water bath $\left(37^{\circ} \mathrm{C}\right)$ and combined with $10 \mathrm{ml}$ warm media in $15 \mathrm{ml}$ clinical centrifuge tubes. The tubes were spun at $258 \mathrm{~g}$ for $3 \mathrm{~min}$, the supernatant was discarded and the pellet then resuspended in $10 \mathrm{ml}$ media in a closed flask and incubated at $37{ }^{\circ} \mathrm{C}, 5 \% \mathrm{CO}_{2}$. The cell suspension was removed from the flask and transferred to a $15 \mathrm{ml}$ centrifuge tube and spun at $258 \mathrm{~g}$ for $3 \mathrm{~min}$. The supernatant was discarded and cells were resuspended in $5 \mathrm{ml}$ media, split and transferred into 2 new flasks and topped with media $(\sim 15 \mathrm{ml})$. Incubation continued at $37^{\circ} \mathrm{C}, 5 \% \mathrm{CO}_{2}$, and this procedure was repeated every $2-3$ days till the desired concentration $\left(5 \times 10^{6}\right.$ cells $\left./ \mathrm{ml}\right)$ of cells was achieved.
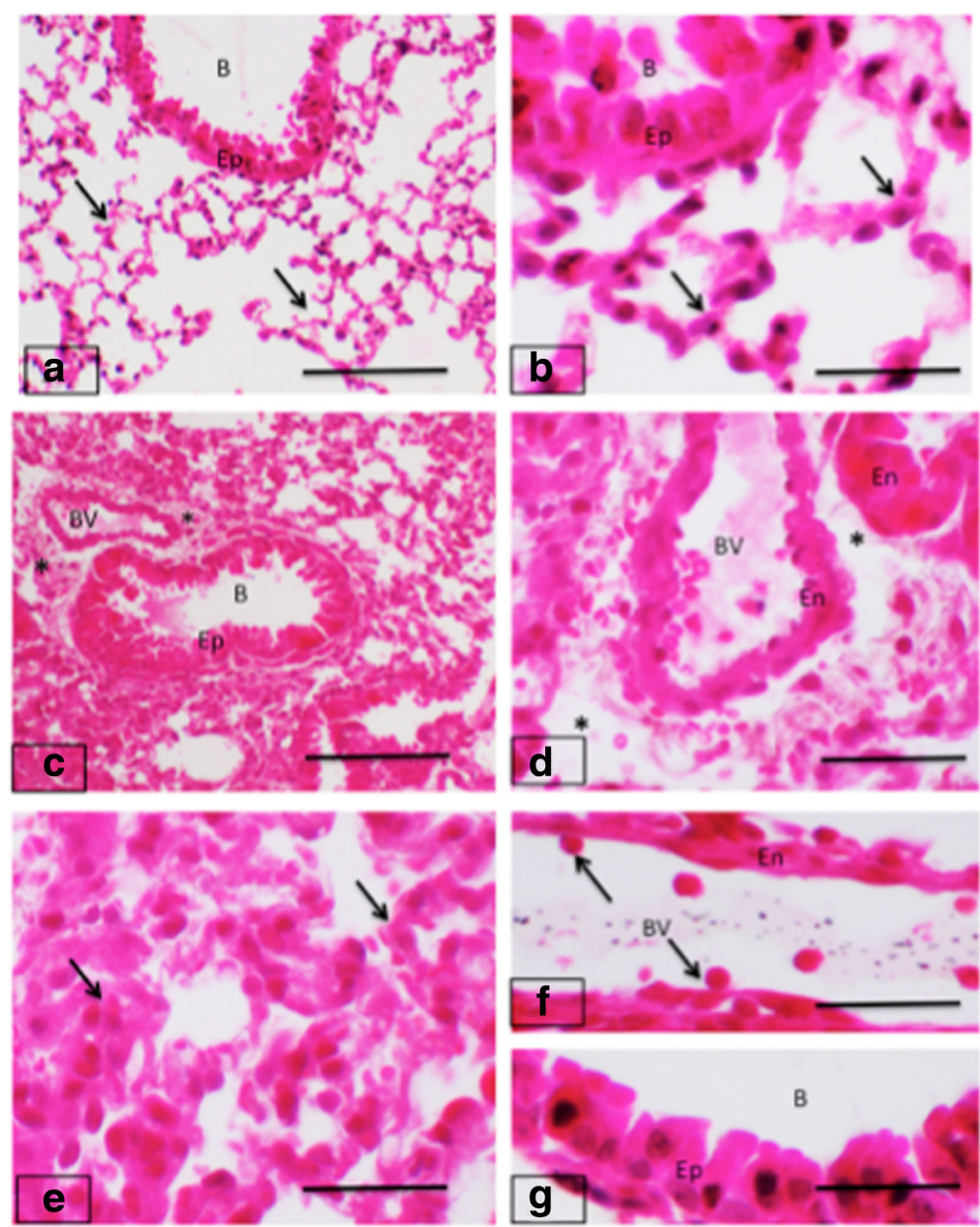

Fig. 1 H\&E staining of mice lungs. Lung sections from control mice (a-b) have normal lung histology of alveolar septa (arrows) and bronchiolar (B) epithelium (Ep). The oral treatment with fipronil caused lung inflammation and lung sections (c-d) show inflammation (asterisks) around bronchioles (B) and blood vessels (BV). Lung sections from mice treated intranasally with fipronil (e-g) show septal congestion in septa (arrows; e)), cells adhering (arrows) to endothelium (En; f) and swollen epithelium (Ep) of bronchioles (B; 1 g). Bar: $100 \mu \mathrm{m}$ 


\section{Fipronil exposure and viability assessment}

Once the cells reached a concentration of $5 \times 10^{5}$ cells $/ \mathrm{ml}$, the cells were incubated with $12 \mathrm{ml}$ media + Phorbol-12myristate-13-acetate (PMA) in 12 well plate for $48 \mathrm{~h}$ to differentiate into macrophages. The cells were treated with various concentrations of fipronil $(0.29 \mu \mathrm{m}$ to $5.72 \mu \mathrm{m}$ per $1 \mathrm{ml}$ ) dissolved in DMSO for $3 \mathrm{~h}$. Following desired incubation, $50 \mu \mathrm{l}$ of $0.25 \%$ Trypsin in Ethylenediaminetetraacetic acid (EDTA) was added to each well in order to facilitate removal of the differentiated macrophages. $150 \mu \mathrm{l}$ of the cells in media were combined with $50 \mu \mathrm{l}$ Trypan blue to assess the viability of cells by trypan blue exclusion method.

\section{Western blots for TLR4 and TLR9}

Following a 3-hour exposure to fipronil, $50 \mu \mathrm{l}$ of $0.25 \%$ Trypsin in EDTA was added to each well for $5 \mathrm{~min}$. Each sample was collected in $1.5 \mathrm{ml}$ centrifuge tubes and spun for $10 \mathrm{~min}$ at $258 \mathrm{~g}$. The supernatant was aspirated off and the pellet was washed with $1 \mathrm{ml}$ Hanks Balanced Salt Solution (HBSS). This step was repeated twice. The supernatant was again aspirated off and one tablet of Protease inhibitor (Roche) was added to $7 \mathrm{ml}$ Radioimmunoprecipitation assay (RIPA) (Sigma-Aldrich) buffer. A $300 \mu \mathrm{l}$ of this RIPA buffer mix was added to each centrifuge tube and vortexed thoroughly. The samples were kept in a $4{ }^{\circ} \mathrm{C}$ refrigerator for $15 \mathrm{~min}$ and vortexed twice during this
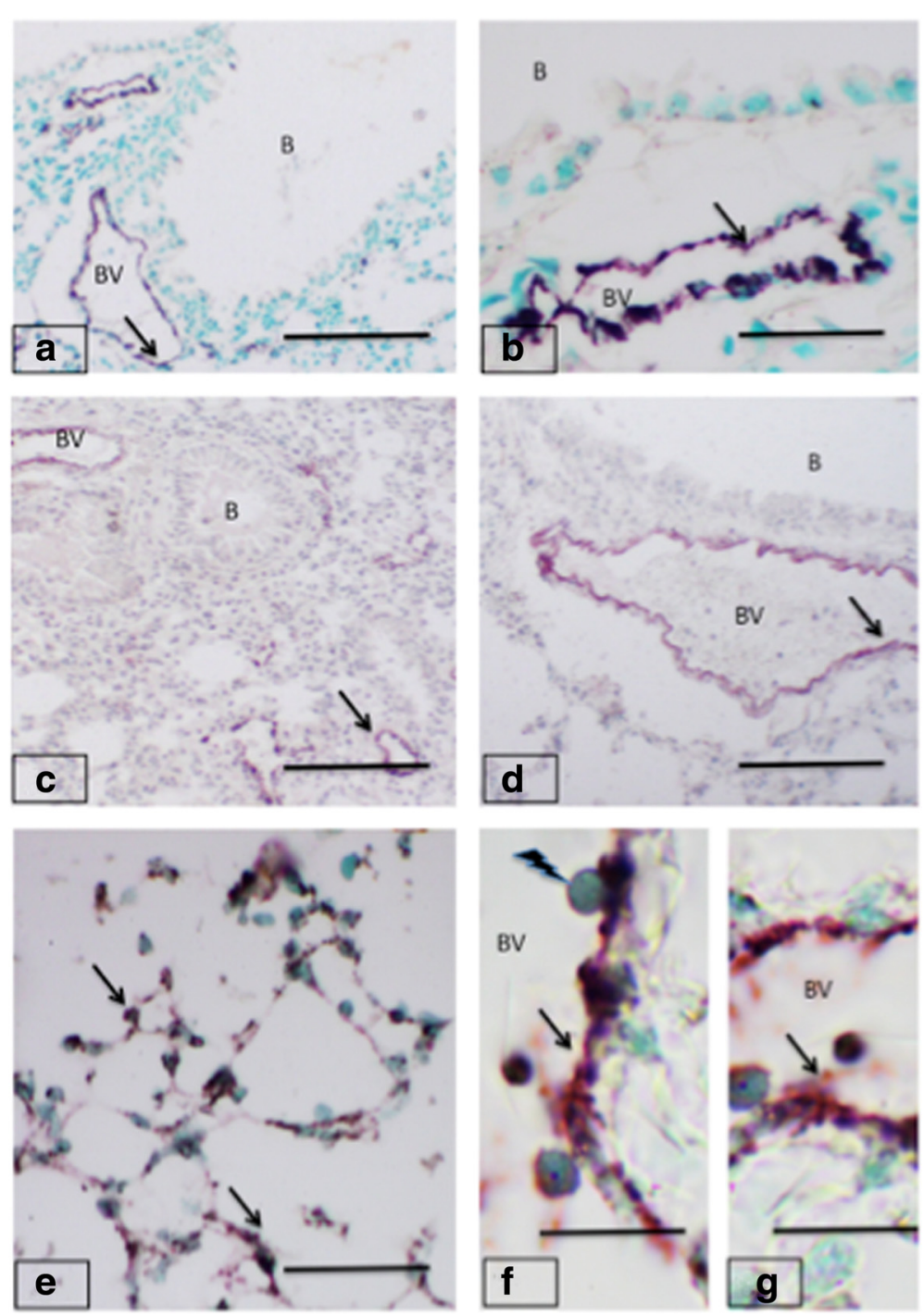

Fig. 2 von Willebrand Factor expression in mice lungs. Lungs from control groups (a-b) show normal vWF staining (arrows) in endothelium of blood vessels (BV) but not in bronchiolar (B) epithelium. The oral treatment with fipronil (c-d) caused inflammation but the expression of VWF (arrows) in blood vessels (BV) remained unchanged. The intranasal fipronil (e-g) showed increased expression (arrows) in alveolar septum (e), endothelium of blood vessels (f-g) as well as the vascular cells. Note vascular cells (lightening arrow) attaching to the endothelium (f). Bar: $100 \mu \mathrm{m}$ 
incubation. The samples were put in the centrifuge at $10,000 \mathrm{~g}$ for $5 \mathrm{~min}$. The supernatant was then carefully removed without disturbing the pellet and stored at $-45^{\circ} \mathrm{C}$ until needed for future assays for up to 1 month. $25 \mu \mathrm{l}$ of protein samples plus indicator were boiled for $5 \mathrm{~min}$ and then loaded into a $12 \%$ SDS-PAGE gel. The proteins were separated via gel electrophoresis at $160 \mathrm{~V}$ for $45-60 \mathrm{~min}$. The gel was collected and placed between 2 sponges, 4 filter papers and Immobilon - FL membrane in a western blot sandwich all previously soaked in the transfer buffer. The protein transfer was performed at $100 \mathrm{~V}$ for $70 \mathrm{~min}$. Following transfer, the membrane was washed in $15 \mathrm{ml}$ PBS and then incubated in $\sim 15 \mathrm{ml}$ blocking buffer (5 \% BSA in PBS) for $1 \mathrm{~h}$. Primary antibodies were mixed with the above blocking buffer with the addition of $0.1 \%$ Tween-20. Primary antibodies (Anti-TLR4, AF1478 R\&D at 1:200 and Anti-TLR9, IMG 305A at 1:200, IMGENEX) were incubated with membranes overnight at $4{ }^{\circ} \mathrm{C}$. Following overnight incubation membranes were washed with PBS and PBST and then incubated with secondary antibody (Goat, anti-mouse Cy5.5 or Donkey Anti-goat Cy3, both at 1:1000, AbCam) in PBS for $30 \mathrm{~min}$. Washing was repeated after this step and then membranes were allowed to dry before visualization using the Typhoon 3 laser fluorescence scanner.

\section{Statistical analyses}

Statistical analysis was performed using statistical software (SPSS, IBM version 21.1 for Windows). For in vivo work a one-way analysis of variance (ANOVA) was run
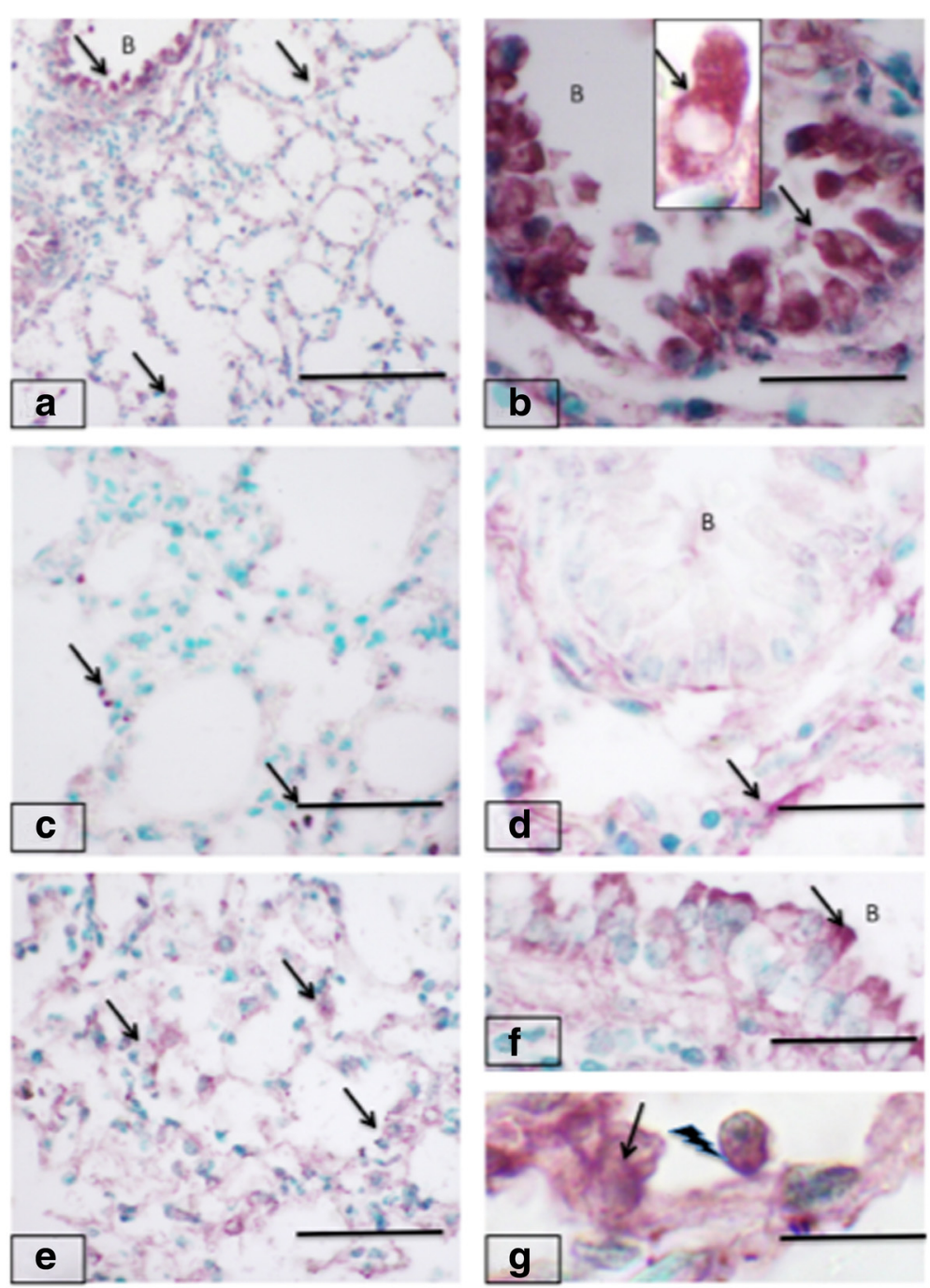

Fig. 3 Toll-like receptor 4 expression in mice lungs. Lungs from control groups (a-b and) show strong staining (arrows) in bronchiolar epithelium (B) and alveolar septum. Note rich cytoplasmic staining in bronchiolar epithelial cells (b and inset). The lungs sections (c-d) from mice treated orally with fipronil show barely minimal staining (arrows) in alveolar septum (c) while it is nearly absent in bronchiolar (B) epithelium. Lung sections from mice exposed to fipronil (e-g) show TLR4 staining similar (arrows) to the control lungs in alveolar septum (e) and epithelium (arrows) of bronchioles (B, $\mathbf{f}$ ). Note TLR4 staining (3 g) in endothelium (arrow) and adhering cells (lightening arrows). Bar $=100 \mu \mathrm{m}$ 
to determine if the number of TLR4/9 positive cells present in the alveolar septa was significantly different between treatments groups. If there was a significant difference, Tukey's Multiple Comparison test was performed to see which treatments differed. For in vitro work, one-way or two-way ANOVA was run to see if there were significant difference between average cell viability. Dunnet's or Tukey's Multiple Comparison Test was ran to see the differences. Since only 2 treatments were used for western blots, Student's Independent $T$-test was used to determine significant differences.

\section{Results}

\section{Hematoxylin and eosin staining}

H\&E stained lung sections from control group showed normal histoarchitecture of lungs except few black spots indicating some evidence of dust particles that the animals could have easily been exposed to throughout the experiment (Fig. 1a and b). Lung sections from animals in the oral fipronil group displayed an accumulation of inflammatory cells around the terminal bronchioles. There was a dilatation of perivascular spaces in lung sections from all the animals. The airway epithelial cells were enlarged and domed in mice treated intranasally with fipronil (Fig. 1c and d). The blood vessel showed a folded appearance. In comparison to the oral group, the intranasal fipronil group displayed overall normal lung architecture and the epithelium did not appear to be activated but there was an increase in accumulation of inflammatory cells in the alveolar septa and the alveoli (Fig. 1e, f and g). Many blood cells were attached to the vascular endothelium (Fig. 1f).
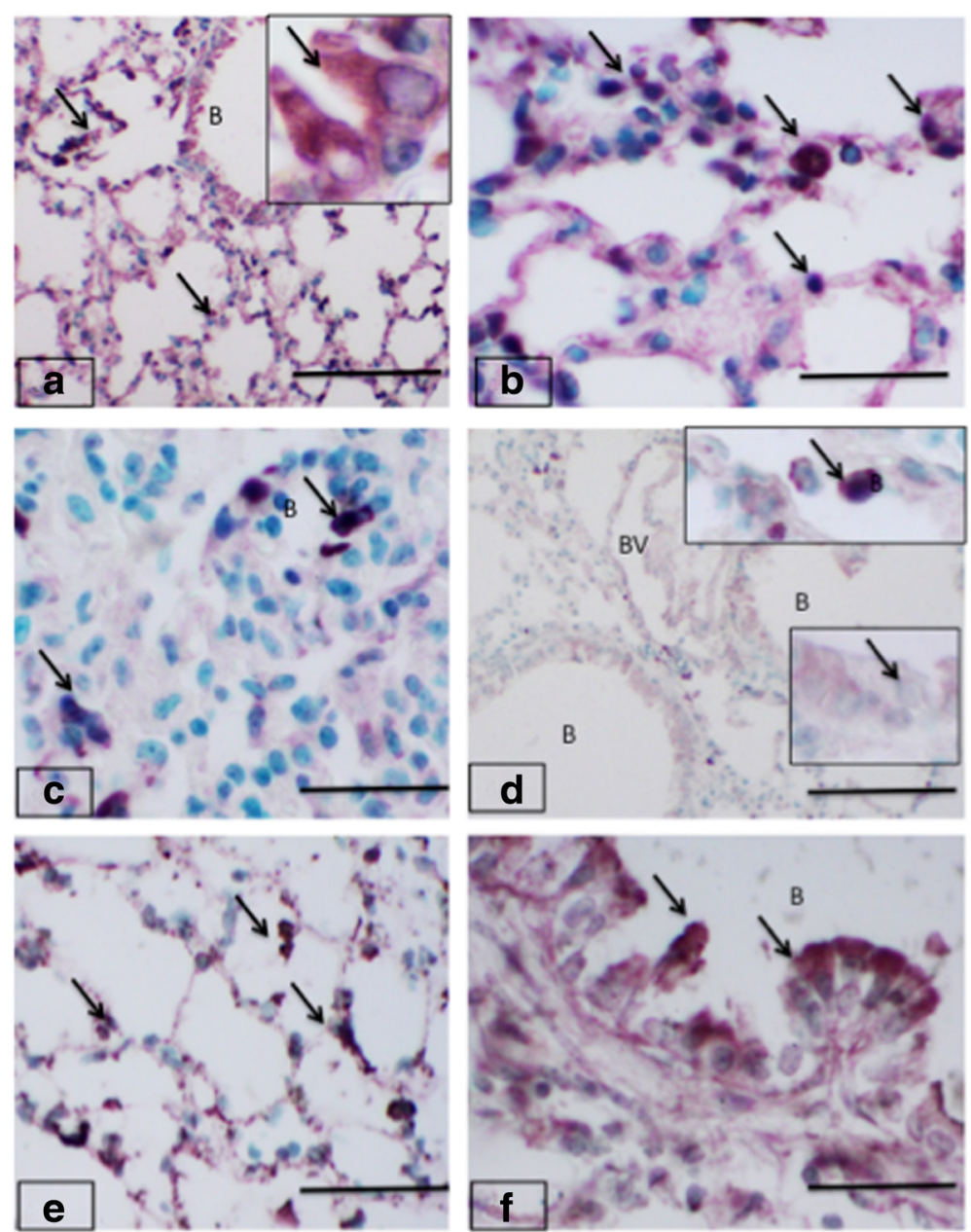

Fig. 4 Toll-like receptor 9 expression in mice lungs. Lungs sections from control groups (a-b) have TLR9 staining (arrows) in alveolar septum (a) and bronchiolar epithelium (a inset). The staining is also seen in the septal cells (arrows) in lung sections from control mice (b). The oral treatment reduced TLR9 staining in lung sections (c-d) and the staining (arrows) was observed in occasional septal cells (c). Bronchiolar epithelium (B; $\mathbf{d}$ and insets) showed much reduced TLR9 staining compared to the controls. Lung sections from mice treated with intranasal fipronil (e-f) showed intense staining (arrows) in alveolar septum (e) and bronchiolar epithelium (f). Bar: $100 \mu \mathrm{m}$ 


\section{Expression of von Willebrand factor}

Lung sections from control animals showed immunopositive staining for vWF in vascular endothelium that was more prominent in larger blood vessels compared to the alveolar septal capillaries (Fig. 2a and b). There was no vWF staining of the bronchiolar epithelium in lung sections from any of the treatment groups. The mice of the oral fipronil group did not have an altered expression of vWF though there was an indication of inflammation in the lung (Fig. 2c and d). An increase in vWF staining was displayed in lung sections from the intranasal fipronil group in areas such as septal capillaries and in the cells accumulated in septal areas (Fig. 2e, $f$ and g). There was specially increased focal vWF staining in endothelial cells and the adhering blood cells (Fig. 2f).

\section{Expression of Toll-like Receptor 4}

The immunopositive TLR4 cells were observed in the cytoplasm of bronchiolar epithelium of oral and intranasal control groups (Fig. 3a and b). Bronchial associated lymphoid tissues (BALT) were present, however indicating the animals were kept in a dusty environment, as BALTs are not normally present in animals kept in sterile environments. The BALTs lacked TLR4 staining. There was reduced staining for TLR4 in lungs of mice administered fipronil orally (Fig. 3c, d). The intranasal fipronil group showed TLR4 staining in alveolar septa, bronchiolar epithelium and vascular endothelium (Fig. 3e, f, g). There reaction was more marked in the apical surface of the airway epithelium (Fig. 3f). The interface of blood cells, vascular endothelium and alveolar macrophages also showed TLR4 staining (Fig. 3g). There was significantly higher number of TLR4 positive cells in the alveolar septa in the intranasal fipronil group compared to intranasal control $(P=0.050)$ and oral fipronil group $(P=0.010)$.

\section{Expression of Toll-like Receptor 9}

TLR9 staining was observed in septa, airway epithelium and blood vessels in the lungs sectins from the oral and intranasal control groups (Fig. 4a, b). Further, TLR9 staining assumed focal appearance in the septa but the airway epithelial staining was considerably reduced in oral fipronil group (Fig. 4c, d). There was minimal TLR9 reaction in vascular endothelium (Fig. 4d). However, TLR9 expression was intense and more prominent in the alveolar septa specially in the large cells in lungs of mice exposed intranasally to fipronil (Fig. 4e). The airway epithelium also showed intense surface staining for TLR9 while the cytoplasmic reaction was reduced in this group compared to control group (Fig. 4f). There was no significant difference for the number of TLR9 positive cells among treatment and control groups.

\section{In vitro results}

\section{Fipronil reduces cell viability}

There was a significant difference in percentage of living cells between the control and both fipronil concentrations. At 3 and $9 \mathrm{~h}$ the low fipronil concentration also had a significantly higher percentage of living cells than the high concentration (Fig. 5). At $24 \mathrm{~h}$ there was not a significant difference in the $\%$ of living cells between the low and high fipronil concentrations (Fig. 6). Further, there was no significant interaction between time vs. concentration $\left(F_{4,120}=11.01, \mathrm{P}=0.115\right)$.

\section{Expression of TLR4 and TLR9}

After the U937 cells were incubated with a high $(5.72 \mu \mathrm{m})$ concentration of fipronil for $3 \mathrm{~h}$, protein extraction was done and western blots were performed for TLR4 and TLR9 followed by densitometric quantification. Figure 7 depicts the average relative density of western blots that were performed three times. The expression of TLR4 and TLR9 did not show any significant difference between the group treated with fipronil vs. the group treated only with DMSO. TLR4 $(P=0.49)$, TLR9 $(P=0.94)$.

\section{Discussion and conclusions}

We report the first in vivo and in vitro effects on lungs and macrophage cells, respectively, of fipronil. The data show lung inflammation following both oral and intranasal treatments with fipronil and an increase in TLR4 positive cells in alveolar septa with intranasal treatment. The in vitro treatment with fipronil caused a concentration dependent reduction in the number of viable U937 macrophage cells but had no effect on the TLR4 or TLR9

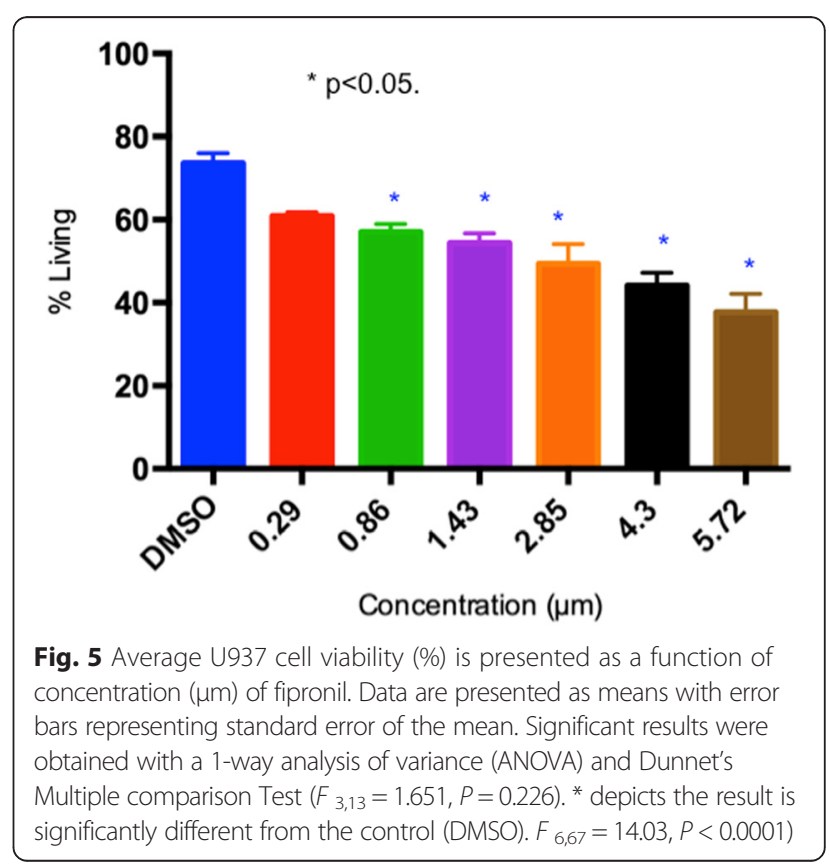




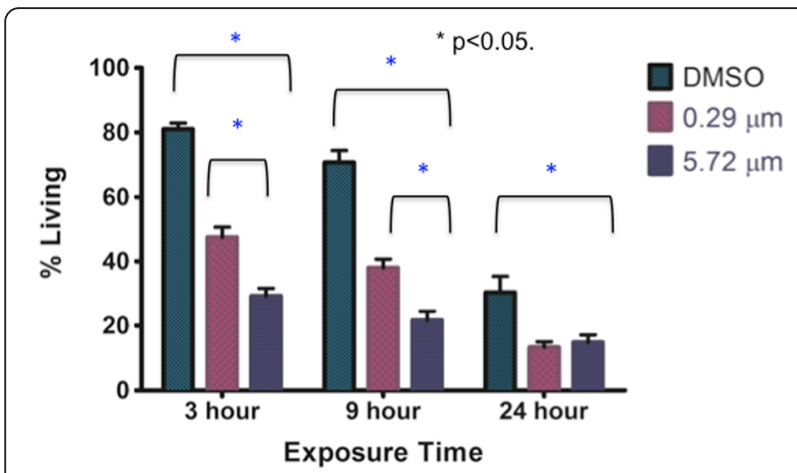

Fig. 6 Average cell viability (\%) as a function of concentration $(\mu \mathrm{m})$. Data are presented as means with error bars representing standard error of the mean. Significant results were obtained via a 2-way ANOVA and Tukey's Multiple Comparison Test. No significant result was found for an interaction between time and concentration $\left(F_{4,120}=11.01\right.$, $P=0.115)$.) There was a signicant result for time $\left(F_{2,120}=29.6, P<0.0001\right)$ and for concentration $\left(F_{2,120}=94.82, P<0.001\right)$ * * represents significant results

expression. Taken together, these data suggest that exposure to fipronil induces lung inflammation and may increase its susceptibility for subsequent endotoxin exposure.

Because of the growing use of fipronil as a pesticide, we examined the effects of oral and intranasal exposures to fipronil on non target organs like lungs. Because this was the first study on pulmonary effects of fipronil, we chose to use fipronil and to focus on any of its metabolite such as sulfone or disulfiny in later studies. Fipronil resulted in lung inflammation in mice as evidenced by accumulation of inflammatory cells, which is considered a hallmark of inflammation [17]. Further, In addition to the routine histology, we also used vWF as a marker of inflammation. the expression of vWF was especially upregaulated in the septal capillaries in mice treated intranasally with fipronil suggesting signs of microvascular inflammation. The expression of vWF in lungs of mice treated orally with fipronil remained changed except some apparent increase in larger pulmonary blood vessels. vWF is a resident adhesive protein in WiebelPalade bodies in endothelial cells, which is exocytosed along with IL-8 and P-selectin by activated endothelial cells $[18,19]$. The data suggest that fipronil given orally or intranasally induced lung inflammation including that in the vasculature.

Lung inflammation is regulated through activation of innate immune system comprised of Toll-like receptors such as TLR4 and TLR9 that bind, respectively, to lipopolysaccharides and CpG molecules [20-22]. We report the first study that fipronil given orally apparently reduced the immunohistochemical expression of TLR4 and TLR9. Intranasal treatment with fipronil increased airway epithelial and vascular endothleial expression of TLR4 and TLR9. The intranasal treatment also increased the number of septal cells expressing TLR4. Previously, the herbicide paraquat given intraperitoneally increased in TLR4 mRNA in the myocardium [23]. Oral treatment with sodium methyldithiocarbamate (SMD), a commonly used pesticide in the U.S., altered expression of TLR4 and inhibited the MAP kinases, which are downstream of TLR4, to reduce the production of proinflammatory cytokines $[24,25]$. Therefore, the observed increase in TLR4 expressing cells in the alveolar septa
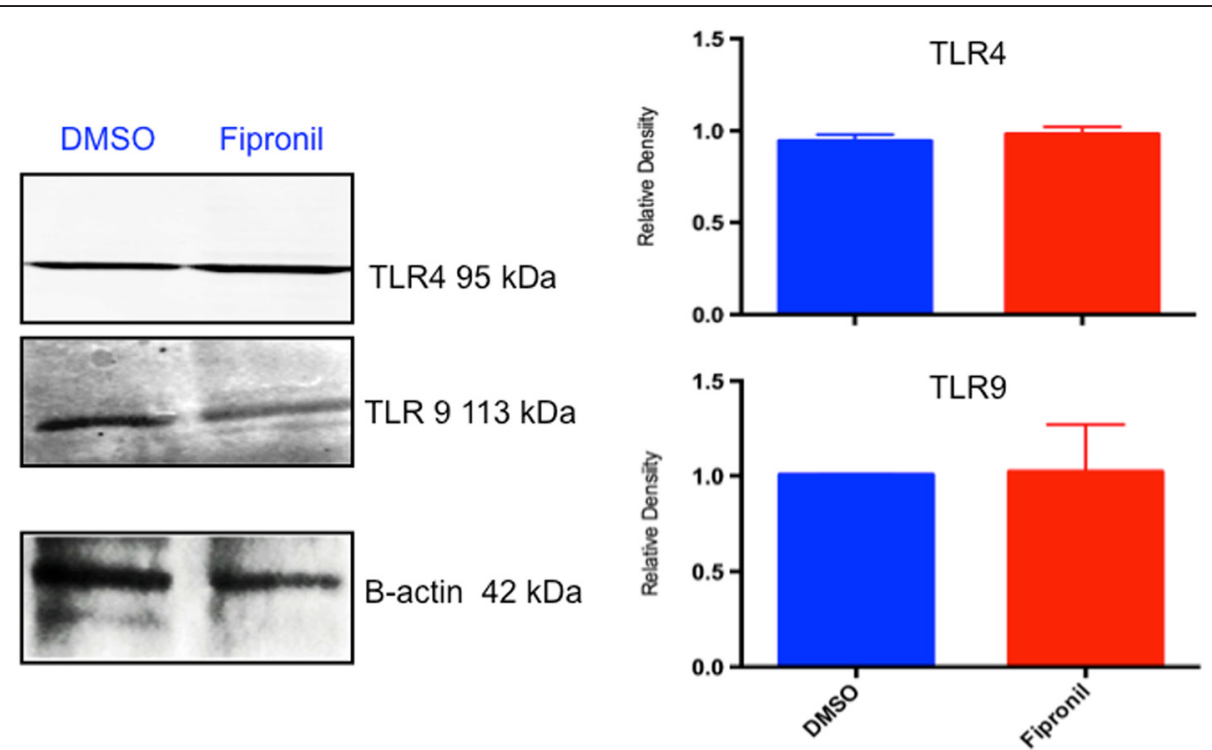

Fig. 7 Western blot of TLR4 and TLR9: Western blots show TLR4 expression cells treated with fipronil and DMSO (5.72 $\mu$ m). The experiment was repeated three times. Densitometry didn't reveal any differences in the expression of TLR4 $(P=0.49)$ and TLR9 $(P=0.94)$ 
would increase susceptibility of animal and humans to endotoxin exposures, which are found in higher amounts in agriculture buildings such as grain elevators and pig barns. Therefore, we need to undertake additional studies where experimental animals exposed to fipronil are challenged with LPS to understand the interactions between the two. The present studies also do not address the reasons for a significant increase in TLR4 positive cells after intranasal but not oral treatment with fipronil. One of the reasons may be the fipronil in the oral group was metabolized and a significantly lesser amount of the original chemical or the metabolite reached the lungs thus activating a fewer number of TLR4 positive cells. Possibly the passage of the chemical or its metabolite(s) through the liver may have attenuated its toxicity for the lung.

We used a macrophage cell line for in vitro studies on the effects of fipronil as many of the septal cells recruited following intranasal treatment are macrophages. Macrophages are central to lung immunity and generation of immune responses [26]. The data showed a reduction in percentages of living cells with a range of concentrations from $0.86 \mu \mathrm{mol}$ to $5.72 \mu \mathrm{mol}$ but not at $0.29 \mu \mathrm{mol}$. We used $0.29 \mu \mathrm{mol}$ and $5.72 \mu \mathrm{mol}$ and found that only the higher concentration caused significant reduction in cell viability at 3 and $9 \mathrm{~h}$ of incubation with fipronil compared to the control and the lower concentration. However, both concentrations reduced cell viability after $24 \mathrm{~h}$ of the exposure. Previously, chlorpyrifos, which is linked to abnormal immune responses in humans induced apoptosis in U937 cells through caspase-3 activation [27]. Our histological observations didn't reveal signs of cell necrosis/apoptosis in lungs following in vivo treatment with fipronil. The in vitro induction of cell death by fipronil may be due to more direct interaction of the pesticide with only one cell type available in the culture, and requires additional studies.

Macrophages express TLR such as TLR4 and TLR9 and use these molecules to sense microbial molecules $[26,28]$. Western blot data showed lack of effect of fipronil on TLR4 and TLR9 expression in macrophage cell line. Because fipronil kills macrophages, the lack of differences in TLR4 and TLR9 expression may be due to increased expression of TLR4 and TLR9/cell to result in lack of differences between the control and the treated macrophage. This would in turn suggest that the expression of TLR4 and TLR9 on live cells may actually be increased as was the case in vivo where the number of septal cells expressing TLR4 was increased following intranasal treatment with fipronil. The preliminary immunofluorescence data on TLR4 expression showed lack of difference between control and fipronil treated cells. However, further studies are needed to clarify the issue.

We report the first in vivo and in vitro effects of fipronil on lungs and macrophage cells, respectively. The data show lung inflammation following both oral and intranasal treatments with fipronil and an increase in TLR4 positive cells in alveolar septa with intranasal treatment. The in vitro treatment with fipronil caused a concentration dependent reduction in the number of viable U937 macrophage cells but had no effect on the TLR4 or TLR9 expression. Taken together, these data suggest that exposure to fipronil induces lung inflammation and may increase its susceptibility for subsequent endotoxin exposure.

\section{Competing interests}

The authors declare they have no competing interests.

\section{Authors' contributions}

KM conceived of the study, analyzed data, and carried out most of the experiments and writing of the manuscript. JPSG and RSS participated in the exposure experiments, data analyses and writing of manuscript. BS assisted with experiment design, writing of paper, and guidance of the project. All authors read and approved the final manuscript.

\section{Acknowledgements}

The work was supported by an International Partnership Project Grant from the University of Saskatchewan Discovery Grant and the Natural Sciences and Engineering Research Council of Canada to Baljit Singh. The work in the laboratories of Dr. JPS Gill and Dr. RS Sethi was supported through funds from the Guru Angad Dev Veterinary and Animal Sciences University, Punjab, India.

\section{Author details \\ ${ }^{1}$ Department of Veterinary Biomedical Sciences, Western College of Veterinary Medicine, University of Saskatchewan, 52 Campus Drive, Saskatoon, SK S7N 5B4, Canada. ${ }^{2}$ School of Animal Biotechnology, Guru Angad Dev Veterinary and Animal Sciences University, Ludhiana, India. ${ }^{3}$ School of Veterinary Public Health, Guru Angad Dev Veterinary and Animal Sciences University, Ludhiana, India.}

Received: 11 December 2015 Accepted: 14 March 2016

Published online: 18 March 2016

\section{References}

1. Kannan K, Tanabe S, Giesy JP, Tatsukawa R. Organochlorine pesticides and polychlorinated biphenyls in foodstuffs from Asian and oceanic countries. Rev Environ Contam Toxicol. 1997;152:1-55.

2. Jennings KA, Canerdy TD, Keller RJ, Atieh BH, Doss RB, Gupta RC. Human exposure to fipronil from dogs treated with frontline. Vet Hum Toxicol. 2002;44(5):301-3.

3. Ratra GS, Erkkila BE, Weiss DS, Casida JE. Unique insecticide specificity of human homomeric rho 1 GABA(C) receptor. Toxicol Lett. 2002;129(1-2):47-53.

4. Ratra GS, Casida JE. GABA receptor subunit composition relative to insecticide potency and selectivity. Toxicol Lett. 2001;122(3):215-22.

5. Ratra GS, Kamita SG, Casida JE. Role of human GABA(A) receptor beta3 subunit in insecticide toxicity. Toxicol Appl Pharmacol. 2001;172(3):233-40. doi:10.1006/taap.2001.9154

6. Zhao X, Yeh JZ, Salgado VL, Narahashi T. Sulfone metabolite of fipronil blocks gamma-aminobutyric acid- and glutamate-activated chloride channels in mammalian and insect neurons. J Pharmacol Exp Ther. 2005;314(1):363-73. doi:10.1124/jpet.104.077891.

7. Badgujar PC, Chandratre GA, Pawar NN, Telang AG, Kurade NP. Fipronil induced oxidative stress involves alterations in SOD1 and catalase gene expression in male mice liver: Protection by vitamins $\mathrm{E}$ and $\mathrm{C}$. Environ Toxicol. 2015. doi:10.1002/tox.22125.

8. Cravedi JP, Delous G, Zalko D, Viguie C, Debrauwer L. Disposition of fipronil in rats. Chemosphere. 2013;93(10):2276-83. doi:10.1016/j.chemosphere.2013. 07.083.

9. Tavares MA, Palma ID, Medeiros HC, Guelfi M, Santana AT, Mingatto FE. Comparative effects of fipronil and its metabolites sulfone and desulfinyl on the isolated rat liver mitochondria. Environ Toxicol Pharmacol. 2015;40(1):206-14. doi:10.1016/j.etap.2015.06.013. 
10. Gill KK, Dumka VK. Biochemical alterations induced by oral subchronic exposure to fipronil, fluoride and their combination in buffalo calves. Environ Toxicol Pharmacol. 2013;36(3):1113-9. doi:10.1016/j.etap.2013.09.011.

11. Gill KK, Dumka VK. Antioxidant status in oral subchronic toxicity of fipronil and fluoride co-exposure in buffalo calves. Toxicol Ind Health. 2013. doi:10.1177/0748233713500376.

12. Zhang $B, X u Z$, Zhang Y, Shao X, Xu X, Cheng J, et al. Fipronil induces apoptosis through caspase-dependent mitochondrial pathways in Drosophila S2 cells. Pestic Biochem Physiol. 2015;119:81-9. doi:10.1016/.jpestbp.2015.01.019.

13. Ki YW, Lee JE, Park JH, Shin IC, Koh HC. Reactive oxygen species and mitogenactivated protein kinase induce apoptotic death of SH-SY5Y cells in response to fipronil. Toxicol Lett. 2012;211(1):18-28. doi:10.1016/j.toxlet.2012.02.022.

14. Lassiter TL, Mackillop EA, Ryde IT, Seidler FJ, Slotkin TA. Is fipronil safer than chlorpyrifos? Comparative developmental neurotoxicity modeled in $\mathrm{PC12}$ cells. Brain Res Bull. 2009;78(6):313-22. doi:10.1016/j.brainresbull.2008.09.020.

15. Caballero MV, Ares I, Martinez M, Martinez-Larranaga MR, Anadon A, Martinez MA. Fipronil induces CYP isoforms in rats. Food Chem Toxicol. 2015;83:215-21. doi:10.1016/j.fct.2015.06.019.

16. de Medeiros HC, Constantin J, Ishii-Iwamoto EL, Mingatto FE. Effect of fipronil on energy metabolism in the perfused rat liver. Toxicol Lett. 2015;236(1):34-42. doi:10.1016/j.toxlet.2015.04.016.

17. Williams $A E$, Chambers RC. The mercurial nature of neutrophils: still an enigma in ARDS? Am J Physiol Lung Cell Mol Physiol. 2014;306(3):L217-30. doi:10.1152/ajplung.00311.2013.

18. Michaux G, Cutler DF. How to roll an endothelial cigar: the biogenesis of Weibel-Palade bodies. Traffic. 2004;5(2):69-78.

19. Hannah MJ, Williams R, Kaur J, Hewlett LJ, Cutler DF. Biogenesis of Weibel-Palade bodies. SeminCell DevBiol. 2002;13:313-24.

20. Ben DF, Yu XY, Ji GY, Zheng DY, Lv KY, Ma B, et al. TLR4 mediates lung injury and inflammation in intestinal ischemia-reperfusion. J Surg Res. 2012;174(2):326-33. doi:10.1016/j.jss.2010.12.005.

21. Aharonson-Raz K, Lohmann KL, Townsend HG, Marques F, Singh B. Pulmonary intravascular macrophages as proinflammatory cells in heaves, an asthma-like equine disease. Am J Physiol Lung Cell Mol Physiol. 2012;303(3):L189-98. doi:10.1152/ajplung.00271.2011.

22. Hoppstadter J, Diesel B, Zarbock R, Breinig T, Monz D, Koch M, et al. Differential cell reaction upon Toll-like receptor 4 and 9 activation in human alveolar and lung interstitial macrophages. Respir Res. 2010;11:124. doi:10.1186/1465-9921-11-124.

23. Dong XS, Xu XY, Sun YQ, Wei L, Jiang ZH, Liu Z. Toll-like receptor 4 is involved in myocardial damage following paraquat poisoning in mice. Toxicology. 2013;312:115-22. doi:10.1016/j.tox.2013.08.009.

24. Tan W, Pruett SB. Effects of sodium methyldithiocarbamate on selected parameters of innate immunity and clearance of bacteria in a mouse model of sepsis. Life Sci. 2015;139:1-7. doi:10.1016/.lfs.2015.08.001.

25. Pruett SB, Zheng Q, Schwab C, Fan R. Sodium methyldithiocarbamate inhibits MAP kinase activation through toll-like receptor 4, alters cytokine production by mouse peritoneal macrophages, and suppresses innate immunity. Toxicol Sci. 2005;87(1):75-85. doi:10.1093/toxsci/kfi215.

26. Schneberger D, Aharonson-Raz K, Singh B. Monocyte and macrophage heterogeneity and Toll-like receptors in the lung. Cell Tissue Res. 2011;343(1):97-106. doi:10.1007/s00441-010-1032-2.

27. Nakadai A, Li Q, Kawada T. Chlorpyrifos induces apoptosis in human monocyte cell line U937. Toxicology. 2006;224(3):202-9. doi:10.1016/j.tox.2006.04.055.

28. Schneberger D, Caldwell S, Kanthan R, Singh B. Expression of Toll-like receptor 9 in mouse and human lungs. J Anat. 2013;222(5):495-503. doi:10.1111/joa.12039.

\section{Submit your next manuscript to BioMed Central and we will help you at every step:}

- We accept pre-submission inquiries

- Our selector tool helps you to find the most relevant journal

- We provide round the clock customer support

- Convenient online submission

- Thorough peer review

- Inclusion in PubMed and all major indexing services

- Maximum visibility for your research

Submit your manuscript at www.biomedcentral.com/submit
Biomed Central 